\title{
The spectral radius of graphs without trees of diameter at most four*
}

\author{
Xinmin $\mathrm{Hou}^{a}$, Boyuan $\mathrm{Liu}^{b}$, Shicheng $\mathrm{Wang}^{c}$ \\ Jun $\mathrm{Gao}^{d}$, Chenhui $\mathrm{Lv}^{e}$ \\ $a, b, c, d, e$ Key Laboratory of Wu Wen-Tsun Mathematics \\ School of Mathematical Sciences \\ University of Science and Technology of China \\ Hefei, Anhui 230026, China.
}

\begin{abstract}
Nikiforov (LAA, 2010) conjectured that for given integer $k$, any graph $G$ of sufficiently large order $n$ with spectral radius $\mu(G) \geq \mu\left(S_{n, k}\right)$ contains all trees of order $2 k+2$, unless $G=S_{n, k}$, where $S_{n, k}=K_{k} \vee \overline{K_{n-k}}$, the join of a complete graph of order $k$ and an empty graph of order $n-k$. In this paper, we show that the conjecture is true for trees of diameter at most four.

Keywords: Brualdi-Solheid-Turán type problem, spectral radius, Erdős-Sós conjecture
\end{abstract}

MSC2010: 05C50, 05C35

\section{Introduction}

In this paper, all graphs considered are simple and finite. For a given graph $G$, let $A(G)$ be the adjacency matrix and let $\mu(G)$ be the largest eigenvalue of $A(G)$, we call $\mu(G)$ the spectral radius of $G$.

As Turán type problems ask for maximum number of edges in graphs of given order not containing a specified family of subgraphs, Brualdi-Solheid-Turán type problems

${ }^{*}$ The work was supported by NNSF of China (No. 11671376) and NNSF of Anhui Province (no. 1708085MA18). 
ask for maximum spectral radius of graphs of given order not containing a specified family of subgraphs. A survey of the Brualdi-Solheid-Turán type problems can be found in [6].

In this paper, we mainly concern a Brualdi-Solheid-Turán type conjecture proposed by Nikiforov [5]. Let $S_{n, k}$ be the graph obtained by joining every vertex of a complete graph of order $k$ to every vertex of an independent set of order $n-k$, that is $S_{n, k}=K_{k} \vee \overline{K_{n-k}}$, the join of $K_{k}$ and $\overline{K_{n-k}}$, and let $S_{n, k}^{+}$be the graph obtained from $S_{n, k}$ by adding a single edge to the independent set of $S_{n, k}$.

Conjecture 1 (Nikiforov, 2010). Let $k \geq 2$ and let $G$ be a graph of sufficiently large order $n$. If $\mu(G) \geq \mu\left(S_{n, k}\right)$, then $G$ contains all trees of order $2 k+2$ unless $G=S_{n, k}$.

The Turán type version of Conjecture 1 is the well-known Erdös-Sós Conjecture [1] which states that every finite simple graph with average degree greater than $k-2$ contains a copy of any tree of order $k$ as a subgraph. The Erdös-Sós Conjecture attracts many attentions and was verified for many specific family of trees, especially for trees of diameter at most four [4].

Nikiforov verified Conjecture 1 for paths [5]. In this paper we show that Conjecture 1 holds for all trees of diameter at most four, here is our main theorem.

Theorem 2. For $k \geq 2$ and $n>2(k+2)^{4}$, every graph $G$ of order $n$ with $\mu(G) \geq$ $\mu\left(S_{n, k}\right)$ contains all trees $T$ of order $2 k+2$ with diam $(T) \leq 4$ as a subgraph, unless $G=S_{n, k}$.

The rest of the paper is arranged as follows. In Section 2, we give some lemmas and notation which will be used in the paper, and the proof of Theorem 2 will be given in Section 3.

\section{Lemmas and notation}

We first give some notation not defined before. Let $G=(V, E)$ be a graph. For $x \in V(G)$, define $N_{G}^{i}(x):=\left\{y: y \in V(G), d_{G}(x, y)=i\right\}$ for $i \geq 1$, where $d_{G}(x, y)$ is the distance between $x$ and $y$ in $G$. In particular, $d_{G}(x):=\left|N_{G}^{1}(x)\right|$ is the degree of $x$ in $G$. Write $\delta(G)$ and $\Delta(G)$ for the minimum and maximum degrees of $G$, respectively. Let $\omega(G)$ and $\operatorname{diam}(G)$ be the number of components and diameter of $G$, respectively. For non-empty subset $S \subseteq V$, write $G[S]$ for the subgraph of $G$ induced by $S$ and $e(G)=|E(G)|$. For $S_{1}, S_{2} \subseteq V(G)$, let $E_{G}\left(S_{1}, S_{2}\right)$ be the set of 
edges of $G$ with one end in $S_{1}$ and the other in $S_{2}$ and write $e_{G}\left(S_{1}, S_{2}\right)=\left|E_{G}\left(S_{1}, S_{2}\right)\right|$. If $S_{1}$ and $S_{2}$ are disjoint, define $G\left[S_{1}, S_{2}\right]$ be the bipartite subgraph with bipartite sets $S_{1}$ and $S_{2}$ and edge set $E_{G}\left(S_{1}, S_{2}\right)$. The subscript $G$ will be omitted if $G$ is clear from the context. We write $[1, n]$ for the set of integers $\{1,2, \ldots, n\}$.

Write $S_{k}$ for a star with $k$ leaves, and we call an isolated vertex a trivial star. A vertex with the maximum degree in a star is called a center of the star. Let $S_{1,2, \ldots, 2}$ be a tree obtained from a star of order $k+2$ by subdividing $k$ edges, also called a spider of order $2 k+2$ with one leg of length one and the others of length two. Write $T_{\ell}\left(\right.$ resp. $\left.T_{\leq \ell}\right)$ for a tree of order $\ell$ (resp. at most $\ell$ ) and define

$$
\mathcal{T}_{\ell}=\left\{T_{\ell}: \operatorname{diam}\left(T_{\ell}\right) \leq 4\right\} \text { and } \mathcal{T}_{\leq \ell}=\left\{T_{\leq \ell}: \operatorname{diam}\left(T_{\leq \ell}\right) \leq 4\right\},
$$

and $\mathcal{T}_{2 k+2}^{*}=\mathcal{T}_{2 k+2} \backslash\left\{S_{1,2, \ldots, 2}\right\}$.

Let $\mathcal{F}$ be a family of graphs. A graph $G$ is said to be $\mathcal{F}$-free if $G$ contains no member of $\mathcal{F}$ as a subgraph. It is well known that, either a tree $T$ has precisely one center (called centered tree), or $T$ has precisely two adjacent centers (called bicentered tree). Furthermore, for a tree $T \in \mathcal{T}_{\leq l}$, the deletion of a center of $T$ reduces a forest with each component a star. In the following proof, a tree $T \in \mathcal{T}_{\leq l}$ always is seen as a rooted tree with root at its center (for a bicentered tree, we choose one of its centers as root such that the number of components is as large as possible in the forest obtained by deleting the root). Let $C_{T}$ (resp. $F_{T}$ ) be the star forest consisting of all stars (resp. all nontrivial stars) by deleting the root of $T$. Then $F_{T} \subseteq C_{T}$ and $C_{T}-F_{T}$ consists of trivial stars (i.e. isolated vertices).

The following matrix theory lemma [3] is the theoretical base of our proof.

Lemma 3 (Lemma 6 in [3]). Given two positive integers $a, b$ and a nonnegative symmetric irreducible matrix $A$ of order $n$, let $\mu$ be the largest eigenvalue of $A$ and let $\mu^{\prime}$ be the largest root of the polynomial $f(x)=x^{2}-a x-b$. Define $B=f(A)$ and let $B_{j}=\sum_{i=1}^{n} B_{i j}$ for $j=1,2, \ldots, n$. If $B_{j} \leq 0$ for all $j=1,2, \ldots, n$, then $\mu \leq \mu^{\prime}$ with equality holds if and only if $B_{j}=0$ for all $j=1,2, \ldots, n$.

Mclennan [4] verified Erdős-Sós conjecture for trees of diameter at most four.

Lemma 4 (Theorem 1 in [4]). Every graph $G$ with $e(G)>\frac{(k-2)|V(G)|}{2}$ contains a $T_{k}$ of diameter at most four.

Lemma 5 (Theorem 4.1 in [2]). Given integers $k \geq 1$ and $n \geq \frac{5 k}{2}$. Let $G$ be a graph of order $n$. If $G$ does not contain a matching of size $k$, then $e(G) \leq e\left(S_{n, k-1}\right)$. The equality holds if and only if $G \cong S_{n, k-1}$. 
We also need the following variant version of Mclennan's result.

Lemma 6. Given $n \geq 2 k+2$. Let $G$ be a graph of order $n$ and $e(G)>\frac{(2 k-1) n}{2}$. If $\Delta(G)=n-1$ then $G$ contains a tree $T \in \mathcal{T}_{\leq 2 k+2}^{*}$.

Proof. Let $T \in \mathcal{T}_{\leq 2 k+2}^{*}$. Then each component of $C_{T}$ is a (trivial or nontrivial) star.

Case 1. $C_{T}$ has a component, say $S_{d}$, of order at least three.

Let $G^{\prime}$ be the graph obtained from $G$ by deleting a vertex $v$ of maximum degree and let $T^{\prime}$ be the tree obtained from $T$ by deleting $V\left(S_{d}\right)$. Then

$$
e\left(G^{\prime}\right)=e(G)-(n-1)>\frac{(2 k-3)(n-1)}{2}
$$

and $\left|V\left(T^{\prime}\right)\right| \leq 2 k-1$. By Lemma 4, $T^{\prime} \subseteq G^{\prime}$. Hence if we embed the center of $S_{d}$ in $v$, then we get an embedding of $T$ in $G$ since $d_{G}(v)=n-1$.

Case 2. Each component of $C_{T}$ has order at most two.

Since $T \neq S_{1,2, \ldots, 2}$, then $\omega\left(F_{T}\right) \leq k-1$. Let $M$ be a maximum matching of $G-v$. Clearly, $m=|M| \leq k-2$, otherwise we can embed $T$ into $G$ centered at $v$ since $d_{G}(v)=n-1$. Assume $M=\left\{a_{i} b_{i}: i \in[1, m]\right\}$. Denote $S=V(G) \backslash(V(M) \cup\{v\})$. By the maximality of $m$, we have $e(G[S])=0$ and $e\left(\left\{a_{i}, b_{i}\right\}, S\right) \leq|S|=n-2 m-1$ for any $i \in[1, m]$. Therefore,

$$
\begin{aligned}
e(G) & =\sum_{i=1}^{m} e\left(\left\{a_{i}, b_{i}\right\}, S\right)+d_{G}(v)+e(G[S])+e(G[M]) \\
& \leq m(n-2 m-1)+(n-1)+m(2 m-1) \\
& =(m+1)(n-2)-1 \\
& <\frac{(2 k-1) n}{2}
\end{aligned}
$$

a contradiction, where the last inequality holds since $m \leq k-2$.

Lemma 7. Given $n \geq 2 k+2$ and let $G$ be a graph of order $n$. If $S_{n, k}$ is a subgraph of $G$, then $G$ contains a $T_{2 k+2}$, unless $G=S_{n, k}$.

Proof. Suppose $G \neq S_{n, k}$. Since $S_{n, k}$ is a spanning subgraph of $G$ and $n \geq 2 k+2$, we have $S_{2 k+2, k}^{+} \subseteq G$. Let $H$ be a copy of $S_{2 k+2, k}^{+}$in $G$. Write $V(H)=\left\{x_{0}, x_{1}, \ldots, x_{2 k+1}\right\}$ such that $H\left[\left\{x_{2}, \ldots, x_{k+1}\right\}\right] \cong K_{k}$ and $H\left[\left\{x_{0}, x_{1}, x_{k+2}, \ldots, x_{2 k+1}\right\}\right] \cong \overline{K_{k+2}}+x_{0} x_{1}$. 
Let $V(T)=A \cup B$ with $|A| \leq|B|$ be the (unique) bipartition of $T$. If $|A| \leq k$, then one can embed $T$ into $H$ since $T$ is a subgraph of the complete bipartite graph $K_{|A|, 2 k+2-|A|}$ and $K_{|A|, 2 k+2-|A|}$ is a subgraph of $H$ for any $1 \leq|A| \leq k$. Now Assume that $|A|=|B|=k+1$. Since $e(T)=2 k+1$, there exists at least one leaf, namely $u$, in $A$. Let $v$ be its neighbour in $B$. Then one can get an embedding of $T$ into $H$ by first embedding $u$ and $v$ into $x_{0}$ and $x_{1}$, respectively, and $A \backslash\{u\}$ and $B \backslash\{v\}$ into $\left\{x_{2}, \ldots, x_{k+1}\right\}$ and $\left\{x_{k+2}, \ldots, x_{2 k+1}\right\}$, respectively.

\section{Proof of Theorem 2}

Note that $\mu=\mu\left(S_{n, k}\right)$ is the largest root of the polynomial

$$
f(x)=x^{2}-(k-1) x-k(n-k) .
$$

Given a graph $G$ of order $n$, let $A=A(G)$ be the adjacent matrix of $G$ and let $B=f(A)$. For $u \in V(G)$, let $B_{u}=\sum_{1 \leq i \leq n} B_{i u}$.

Now assume $G$ is a $\mathcal{T}_{2 k+2}$-free graph on $n$ vertices with $\mu(G) \geq \mu\left(S_{n, k}\right)$ and $G \neq$ $S_{n, k}$. By Lemma 3, there must exist a vertex $u \in V(G)$ such that $B_{u} \geq 0$.

Claim 1. Every vertex $u \in V(G)$ with $B_{u} \geq 0$ has degree at least $k+1$.

Proof of Claim 1: For any $v \in V(G)$, define $L=L_{v}$ be the graph with vertex set $V(L)=N^{1}(v) \cup N^{2}(v)$ and edge set $E(L)=E\left(N^{1}(v)\right) \cup E\left(N^{1}(v), N^{2}(v)\right)$. By the definition of $B$, for any $v \in V(G)$, we have

$$
B_{v}=\sum_{x \in N^{1}(v)} d_{L}(x)-(k-2) d_{G}(v)-k(n-k) .
$$

Since $\left|N^{1}(v)\right|=d_{G}(v)$ and $d_{L}(x) \leq n-2$ for any $x \in N^{1}(v)$, we have

$$
B_{v} \leq(n-2) d_{G}(v)-(k-2) d_{G}(v)-k(n-k)=\left(d_{G}(v)-k\right)(n-k) .
$$

If $B_{v} \geq 0$ then $d_{G}(v) \geq k$. If $d_{G}(v) \geq k+1$ then we are done. Now assume $d_{G}(v)=k$. Then $B_{v}=0$. By (2), we have $L \cong S_{n-1, k}$, or equivalently, $S_{n, k}$ is a subgraph of $G$. By Lemma 7, either $G=S_{n, k}$ or $G$ contains a $T_{2 k+2}$, a contradiction to the assumption. 
Now let $u \in V(G)$ be a vertex with $B_{u} \geq 0$. Then $d_{G}(u) \geq k+1$ by Claim 1. Let $T \in \mathcal{T}_{2 k+2}$. Let $p=\omega\left(C_{T}\right)$ and $p^{\prime}=\omega\left(F_{T}\right)$. Then $p^{\prime} \leq e\left(F_{T}\right)=2 k+1-p$. Thus $p^{\prime} \leq k$ since $p^{\prime} \leq p \leq 2 k+1$.

Case 1. $d_{G}(u)<p$.

In this case, we show that $T$ can be embedded in $G$ rooted at some vertex of $N^{1}(u)$, so we get a contradiction. For each $x \in N^{1}(u)$, let

$$
C(x)=\left\{y \in N^{2}(u): d_{L}(y) \geq 2 k+2-p\right\} .
$$

Claim 2. There exists some vertex $x \in N^{1}(u)$ such that $|C(x)| \geq p^{\prime}$ and $\mid N^{1}(x) \cap$ $N^{2}(u) \mid \geq p$.

Proof of Claim 圆: Suppose to the contrary that for any vertex $x \in N^{1}(u)$, we have $|C(x)| \leq p^{\prime}-1$. Hence

$$
\begin{aligned}
& \sum_{x \in N^{1}(u)} \sum_{y \in N^{1}(x) \cap N^{2}(u)} d_{L}(y) \\
& \leq\left|N^{1}(u)\right|\left[\left(p^{\prime}-1\right)\left|N^{1}(u)\right|+\left(n-1-\left|N^{1}(u)\right|-p^{\prime}+1\right)(2 k+1-p)\right] \\
& =d_{G}(u)\left[(2 k+1-p) n-\left(2 k+1-p-p^{\prime}+1\right) d_{G}(u)-p^{\prime}(2 k+1-p)\right] \\
& \leq d_{G}(u)(2 k+1-p) n \\
& \leq d_{G}(u)\left(2 k-d_{G}(u)\right) n \\
& \leq\left(k^{2}-1\right) n,
\end{aligned}
$$

the last inequality holds since $d_{G}(u) \geq k+1>k$.

On the other hand,

$$
\begin{aligned}
& \sum_{x \in N^{1}(u)} \sum_{y \in N^{1}(x) \cap N^{2}(u)} d_{L}(y)=\sum_{y \in N^{2}(u)} d_{L}^{2}(y) \\
\geq & \frac{1}{\left|N^{2}(u)\right|}\left(\sum_{y \in N^{2}(u)} d_{L}(y)\right)^{2} \\
\geq & \frac{1}{\left|N^{2}(u)\right|}\left(\sum_{x \in N^{1}(u)} d_{L}(x)-\left|N^{1}(u)\right|\left(\left|N^{1}(u)\right|-1\right)\right)^{2} \\
> & \frac{1}{n}\left(B_{u}+(k-2) d_{G}(u)+k(n-k)-d_{G}(u)\left(d_{G}(u)-1\right)\right)^{2} \\
> & \frac{1}{n}\left(k n-6 k^{2}\right)^{2},
\end{aligned}
$$


the first inequality holds by Cauchy-Schwartz inequality, the second inequality holds since $N^{2}(u)$ is an independent set of $L$, and the last inequality holds since $k+1 \leq$ $d_{G}(u) \leq p \leq 2 k+1$. By (3) and (4), we have $\frac{\left(k n-6 k^{2}\right)^{2}}{n}<\left(k^{2}-1\right) n$, that is $n<$ $\frac{6 k^{2}}{k-\sqrt{k^{2}-1}}<12 k^{3}$, a contradiction to $n \geq 2(k+2)^{4}$. Hence, there is at least one vertex $x \in N^{1}(u)$ with $|C(x)| \geq p^{\prime}$, without loss of generality, assume $x$ is such a vertex in $N^{1}(u)$ which maximizes the value of the summation $\sum_{y \in N^{1}(x) \cap N^{2}(u)} d_{L}(y)$. Note that $d_{L}(y) \leq\left|N^{1}(u)\right|=d_{G}(u)$. By (4), we have

$$
\left|N^{1}(x) \cap N^{2}(u)\right|>\frac{1}{d_{G}^{2}(u)} \frac{\left(k n-6 k^{2}\right)^{2}}{n} \geq \frac{\left(k n-6 k^{2}\right)^{2}}{9 k^{2} n}>\frac{n}{9}-2 k>p .
$$

This completes the proof of the claim.

By Claim 2, we can choose a vertex $x \in N^{1}(u)$ with $|C(x)| \geq p^{\prime}$ and $\mid N^{1}(x) \cap$ $N^{2}(u) \mid \geq p$. Note that for any vertex $y \in C(x), d_{L}(y) \geq 2 k+2-p$. Hence $d_{L-x}(y) \geq$ $2 k+1-p$ for any $y \in C(x)$. Therefore, we can greedily embed the star forest $F_{T}$ into $L-x$ with centers in $C(x)$. This completes the proof of this case.

Case 2. $p \leq d_{G}(u) \leq 2(k+2)^{3}$.

If we find a copy $F$ of $F_{T}$ in $L=L_{u}$ with its centers in $N^{1}(u)$ then the subgraph induced by $V(F) \cup\{u\}$ contains a copy of $T$ rooted at $u$, also a contradiction to the assumption. In the following, we will show that we truly can find such an $F_{T}$ in $L$.

Let $C=\left\{x \in N^{1}(u): d_{L}(x) \geq 2 k\right\}$. If $|C| \geq p^{\prime}$, then we can greedily embed $F_{T}$ into $L$ with its centers in $C$. Hence it is sufficient to show that such a subset $C$ exists.

Claim 3. We have $|C| \geq k \geq p^{\prime}$.

Proof of Claim 3: Suppose to the contrary that $|C| \leq k-1$. Then

$$
\begin{aligned}
B_{u} & =\sum_{x \in N^{1}(u)} d_{L}(x)-(k-2) d_{G}(u)-k(n-k) \\
& \leq(k-1)(n-2)+\left(d_{G}(u)-k+1\right) \cdot 2 k-(k-2) d_{G}(u)-k(n-k) \\
& =-n+(k+2) d_{G}(u)-k^{2}+2 \\
& \leq-n+2(k+2)^{4}-k^{2}+2<0,
\end{aligned}
$$

a contradiction. The proof of the case is completed. 
Case 3. $d_{G}(u)>2(k+2)^{3}$.

Let $L=L_{u}$ be defined the same as in Claim 1, Recall that

$$
B_{u}=\sum_{x \in N^{1}(u)} d_{L}(x)-(k-2) d_{G}(u)-k(n-k) \geq 0
$$

we have $\sum_{x \in N^{1}(u)} d_{L}(x) \geq(k-2) d_{G}(u)+k(n-k)$.

If $T=S_{1,2, \ldots, 2}$, then both $L$ and $G\left[N^{1}(u)\right]$ contain no matching of size $k$ (otherwise we have an embedding of $T$ rooted at $u)$. Hence Lemma 5 implies that $e\left(G\left[N^{1}(u)\right]\right) \leq$ $e\left(S_{\left|N^{1}(u)\right|, k-1}\right)$ and $e(L) \leq e\left(S_{\left|N^{1}(u)\right|+\left|N^{2}(u)\right|, k-1}\right)$. So we have

$$
\begin{aligned}
\sum_{x \in N^{1}(u)} d_{L}(x) & =e\left(G\left[N^{1}(u)\right]\right)+e(L) \\
& \leq e\left(S_{\left|N^{1}(u)\right|, k-1}\right)+e\left(S_{\left|N^{1}(u)\right|+\left|N^{2}(u)\right|, k-1}\right) \\
& =2\left(d_{G}(u)-k+1\right)(k-1)+(k-1)(k-2)+(k-1)\left|N^{2}(u)\right| \\
& \leq(k-2) d_{G}(u)+k(n-k)-\left|N^{2}(u)\right| .
\end{aligned}
$$

On the other hand, we have $\sum_{x \in N^{1}(u)} d_{L}(x) \geq(k-2) d_{G}(u)+k(n-k)$. This implies that $N^{2}(u)=\emptyset$ and $L=G\left[N^{1}(u)\right] \cong S_{n-1, k-1}$, and hence $G$ contains a $S_{n, k}$ as a subgraph. Lemma 7 implies that any tree $T_{2 k+2}$ (including $S_{1,2, \ldots, 2}$ ) is a subgraph of $G$ since $G \neq S_{n, k}$, a contradiction.

Now assume that $T \in T_{2 k+2}^{*}$.

Subase 3.1. $e\left(N^{1}(u), N^{2}(u)\right)>k d_{G}(u)+2 k\left(\left|N^{2}(u)\right|+1\right)-k(n-k)$.

Let $G_{1}$ be the graph with vertex set $\{u\} \cup V(L)$ and edge set $E\left(u, N^{1}(u)\right) \cup E(L)$. Then we have

$$
\begin{aligned}
e\left(G_{1}\right) & =d_{G}(u)+\frac{1}{2}\left(\sum_{x \in N^{1}(u)} d_{L}(x)+e\left(N^{1}(u), N^{2}(u)\right)\right) \\
& >d_{G}(u)+\frac{(k-2) d_{G}(u)+k(n-k)+k d_{G}(u)+2 k\left(\left|N^{2}(u)\right|+1\right)-k(n-k)}{2} \\
& =k\left(d_{G}(u)+\left|N^{2}(u)\right|+1\right) \\
& =k\left|V\left(G_{1}\right)\right|
\end{aligned}
$$

By Lemma 4, $G_{1}$ contains a $T_{2 k+2}$ of diameter at most 4 , a contradiction.

Subcase 3.2. $e\left(N^{1}(u), N^{2}(u)\right) \leq k d_{G}(u)+2 k\left(\left|N^{2}(u)\right|+1\right)-k(n-k)$. 
Let $G_{2}$ be the graph with vertex set $\{u\} \cup N^{1}(u)$ and edge set $E\left(u, N^{1}(u)\right) \cup E\left(N^{1}(u)\right)$. Then we have

$$
\begin{aligned}
e\left(G_{2}\right) & =d_{G}(u)+\frac{1}{2}\left(\sum_{x \in N^{1}(u)} d_{L}(x)-\left|E\left(N^{1}(u), N^{2}(u)\right)\right|\right) \\
& \geq d_{G}(u)+\frac{(k-2) d_{G}(u)+k(n-k)-k d_{G}(u)-2 k\left(\left|N^{2}(u)\right|+1\right)+k(n-k)}{2} \\
& =k\left(n-\left|N^{2}(u)\right|\right)-k(k+1) \\
& \geq k\left(d_{G}(u)+1\right)-k(k+1) \\
& >\frac{(2 k-1)\left(d_{G}(u)+1\right)}{2} \\
& =\frac{(2 k-1)\left|V\left(G_{2}\right)\right|}{2},
\end{aligned}
$$

the last inequality holds since $d_{G}(u)>2(k+2)^{3}$.

Note that $\Delta\left(G_{2}\right)=d_{G}(u)=\left|V\left(G_{2}\right)\right|-1$. By Lemma 6, $G_{2}$ contains a $T_{2 k+2} \in$ $\mathcal{T}_{2 k+2}^{*}$, a contradiction.

The proof is completed.

\section{Concluding remarks}

In fact, Nikiforov's conjecture has two parts.

Conjecture 8 (Conjecture 16 in [5]). Let $k \geq 2$ and let $G$ be a graph of sufficiently large order $n$.

(a) if $\mu(G) \geq \mu\left(S_{n, k}\right)$, then $G$ contains all trees of order $2 k+2$ unless $G=S_{n, k}$;

(b) if $\mu(G) \geq \mu\left(S_{n, k}^{+}\right)$, then $G$ contains all trees of order $2 k+3$ unless $G=S_{n, k}^{+}$.

In the paper, we prove that (a) is true for all trees $T_{2 k+2} \in \mathcal{T}_{2 k+2}$, and $S_{n, k}$ is the unique extremal graph with maximum spectral radius among all of the $\mathcal{T}_{2 k+2}$-free graphs of order $n$. Let $S_{2, \ldots, 2}$ be the spider of order $2 k+3$ with each leg of length two and let $\mathcal{T}_{2 k+3}^{*}=\mathcal{T}_{2 k+3} \backslash\left\{S_{2, \ldots, 2}\right\}$. We believe that, for sufficiently large integer $n$, (b) is true for all trees $T_{2 k+3} \in \mathcal{T}_{2 k+3}$, moreover, $S_{n, k}$ is the unique extremal graph for $\mathcal{T}_{2 k+3}^{*}$ and $S_{n, k}^{+}$for $S_{2, \ldots, 2}$. We leave this as a problem. 


\section{References}

[1] P. Erdős, Some problems in graph theory, Theory of Graphs and Its Applications, M. Fiedler, Editor, Academic Press, New York, 1965, pp. 29-36.

[2] P. Erdös, T. Gallai, On maximal paths and circuits of graphs, Acta Math. Acad. Sci. Hungar. 10 (1959) 337356.

[3] J. Gao, X. Hou, The spectral radius of graphs without long cycles, submitted.

[4] A. McLennan, The Erdős-Sós Conjecture for trees of diameter four. J. Graph Theory, 2005, 49(4): 291-301.

[5] V. Nikiforov, The spectral radius of graphs without paths and cycles of specified length. Linear Algebra Appl., 2010, 432(9): 2243-2256.

[6] V. Nikiforov, Some new results in extremal graph theory. Surveys in Combinatorics 2011, Cambridge University Press, 2011, 141-181.

[7] W. Yuan, B. Wang, M. Zhai, On the spectral radii of graphs without given cycles. Electron J. Linear Algebra, 2012, 23(1): 43.

[8] M, Zhai, B. Wang, Proof of a conjecture on the spectral radius of $C_{4}$-free graphs. Linear Algebra Appl., 2012, 437(7): 1641-1647. 